PROCEEDINGS OF THE

AMERICAN MATHEMATICAL SOCIETY

Volume 135, Number 1, January 2007, Pages 269-276

S 0002-9939(06)08449-8

Article electronically published on July 28, 2006

\title{
A FAST ALGORITHM TO COMPUTE THE $\pi$-LINE THROUGH POINTS INSIDE A HELIX CYLINDER
}

STEVEN H. IZEN

(Communicated by M. Gregory Forest)

\begin{abstract}
In the context of helical cone-beam CT, Danielsson et al. discovered that for each point interior to the cylindrical surface containing a given helix, there is exactly one line segment passing through the point which intersects two points less than one turn apart on the helix. This segment is called a $\pi$-line. A new constructive algebraic proof of this result is presented along with a fast algorithm to compute the endpoints of the $\pi$-line through an arbitrary point in the interior of the helix cylinder. This proof exposes the geometry of the decomposition of a cylinder interior as a disjoint union of $\pi$-lines.
\end{abstract}

\section{INTRODUCTION}

In helical cone-beam tomography, a radiation source moves on a helical path surrounding a cylindrical volume of interest. For each point on the source helix, projection data are collected for a set of lines emanating from the source, passing through the scan cylinder. In one class of reconstruction algorithms, the projection data are first processed, and then backprojected into the scan region. That is, for each source position, processed projection data from lines passing through a point in the scan region are summed to give the reconstruction at that point.

Several algorithms of this class [1, 2, 3, 4, exploit a surprising geometric relationship between a helix and points which are interior to the cylinder on which the helix lies. Namely, for each point interior to the helix cylinder there is exactly one line segment passing through the point which intersects two points of the helix less than one turn apart. The existence of this line segment, called the $\pi$-line, was discovered by Danielsson et al. 11 who provided a geometric proof of its existence and uniqueness. An algebraic proof was later given by Defrise et al. 3. The purpose of this article is to present a new, constructive, algebraic proof of the existence and uniqueness of a $\pi$-line. Moreover, from the proof, geometric insight is obtained on the decomposition of the scan cylinder volume as a disjoint union of $\pi$-lines. Also arising from the proof is a fast algorithm for the computation of the endpoints of the $\pi$-line through a specified point in the scan cylinder volume.

Received by the editors February 26, 2004 and, in revised form, July 25, 2005.

2000 Mathematics Subject Classification. Primary 65H05; Secondary 51N05, 65R10. 


\section{DeCOMPOSITION OF THE SCAN CYLINDER AS $\pi$-LINES}

Consider a helix $H$ of radius $R$ and pitch $h$ with axis in the $z$ direction. Without loss of generality assume $H$ passes through $(R, 0,0)$. Let $\boldsymbol{a}(\lambda)$ denote the point

$$
\boldsymbol{a}(\lambda)=(R \cos \lambda, R \sin \lambda, h \lambda)
$$

on the helix. The support of the object to be imaged is assumed to be inside the scan cylinder volume $S$ of radius $r<R$, also with axis in the $z$ direction:

$$
S=\left\{(x, y, z) \in \mathbb{R}^{3} \mid \sqrt{x^{2}+y^{2}} \leq r\right\} .
$$

Definition 1. A $\pi$-line $\Pi\left(\lambda_{1}, \lambda_{2}\right)$ is a line segment between two points $\boldsymbol{a}\left(\lambda_{1}\right), \boldsymbol{a}\left(\lambda_{2}\right)$ $\in H$, with $0<\lambda_{2}-\lambda_{1}<2 \pi$.

Theorem 1. Let $H$ and $S$ be as above. Then, any point $(x, y, z) \in S$ is on a unique $\pi$-line.

Theorem 1 will be proved by decomposing the scan cylinder into a family of chips, each chip being a subfamily of $\pi$-lines. Once a point is known to reside on a particular chip, the endpoints of the $\pi$-line containing the point are easily identified. The correct chip is identified by solving a transcendental equation. It will be shown that this transcendental equation admits a unique solution. Moreover, Newton's method converges at least quadratically to that solution, leading to a practical algorithm for the computation of the $\pi$-line endpoints.

Definition 2. The chip anchored at $\boldsymbol{a}(\lambda)$, denoted by $C(\lambda)$, is the portion within the scan cylinder $S$ of the union of all $\pi$-lines with endpoints equidistant along the helix from $\boldsymbol{a}(\lambda)$. That is,

$$
C(\lambda)=S \cap\left\{\boldsymbol{x} \in \mathbb{R}^{3} \mid \boldsymbol{x} \in \Pi(\lambda-\alpha, \lambda+\alpha), \alpha \in(0, \pi)\right\} .
$$

The chip axis is the line segment on the chip along the line connecting $(0,0, h \lambda)$ to $\boldsymbol{a}(\lambda)$.

It should be noted that $C(\lambda \pm \pi / 2)$ are identical to the upper and lower $\pi$-surfaces introduced in [2].

It is clear from the definition that the chip is a disjoint union of $\pi$-lines. In particular, each point on the chip is on exactly one of the constituent $\pi$-lines. See Figure 1.

Lemma 2. Every $\pi$-line is on exactly one chip.

Proof. By construction, the $\pi$-line $\Pi\left(\lambda_{1}, \lambda_{2}\right)$ connecting $\boldsymbol{a}\left(\lambda_{1}\right)$ to $\boldsymbol{a}\left(\lambda_{2}\right)$ is on (and can only be on) the chip $C\left(\left(\lambda_{1}+\lambda_{2}\right) / 2\right)$, with $\alpha=\left(\lambda_{2}-\lambda_{1}\right) / 2$.

Consider a point $\boldsymbol{x}=(x, y, z) \in C(0)$. Then

$$
\boldsymbol{x} \in \Pi(-\alpha, \alpha),
$$

where

$$
\alpha=\cos ^{-1}(x / R) .
$$

The $z$ coordinate of $\boldsymbol{x}$ is computed to be

$$
z=\frac{h y \cos ^{-1}(x / R)}{\sqrt{R^{2}-x^{2}}} .
$$




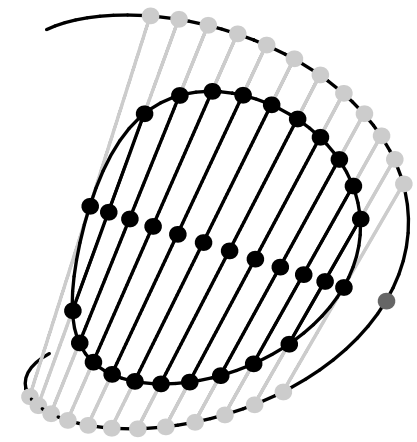

Figure 1. The chip $C(\lambda)$ is generated by the union of the family of $\pi$-lines which connect points of the helix equidistant from the anchor point $\boldsymbol{a}(\lambda)$. Only the portion of the $\pi$-lines inside the scan cylinder (shown in black) contribute. The anchor point for the chip is shown in dark gray, and the mid-points of the $\pi$-lines are on the chip axis. For the chip shown, $r / R=3 / 4$, and $h=1 / 3$.

Thus, $C(0)$ is parametrized in Cartesian coordinates by

$$
C(0)=\left\{\left(x, y, \frac{h y \cos ^{-1} x / R}{\sqrt{R^{2}-x^{2}}}\right) \mid \sqrt{x^{2}+y^{2}} \leq r\right\} .
$$

Introduce polar coordinates $\rho, \theta$ on the $x y$ plane. Then

(8)

$$
C(0)=\left\{\left(\rho \cos \theta, \rho \sin \theta, h \frac{(\rho / R) \sin \theta \cos ^{-1}((\rho / R) \cos \theta)}{\sqrt{1-(\rho / R)^{2} \cos ^{2} \theta}}\right) \mid \begin{array}{c}
0 \leq \rho \leq r, \\
-\pi<\theta \leq \pi
\end{array}\right\} .
$$

Moving the anchor point from $\boldsymbol{a}(0)$ to a general point $\boldsymbol{a}(\lambda)$ rotates the chip in the $x y$ plane by $\lambda$ and translates by $h \lambda$ in the $z$ direction. If $\theta$ is now understood to be the angle in the $x y$ plane measured with respect to the chip axis, then

$$
\begin{aligned}
& C(\lambda)=\{(\rho \cos (\theta+\lambda), \rho \sin (\theta+\lambda), \\
&\left.\left.h\left(\lambda+\frac{(\rho / R) \sin \theta \cos ^{-1}((\rho / R) \cos \theta)}{\sqrt{1-(\rho / R)^{2} \cos ^{2} \theta}}\right)\right) \mid \begin{array}{c}
0 \leq \rho \leq r \\
-\pi<\theta \leq \pi
\end{array}\right\} .
\end{aligned}
$$

To simplify the following algebra, without loss of generality, assume that $R=1$.

Let $\rho, \gamma, z$ be the cylindrical coordinates for $\boldsymbol{x} \in S$. See Figure 2. Then $\gamma$ and $z$ are related to $\lambda$ and $\theta$ by

$$
\begin{aligned}
\gamma & =\lambda+\theta \quad \bmod 2 \pi \\
z / h & =\lambda+\frac{\rho \sin \theta \cos ^{-1}(\rho \cos \theta)}{\sqrt{1-\rho^{2} \cos ^{2} \theta}} .
\end{aligned}
$$

Eliminate $\lambda$ to obtain an equation for $\theta$,

$$
\theta-\frac{\rho \sin \theta \cos ^{-1}(\rho \cos \theta)}{\sqrt{1-\rho^{2} \cos ^{2} \theta}}=\gamma-z / h \bmod 2 \pi .
$$

Next, it is shown that (12) can be solved for $\theta$ as long as $\rho<1$. 


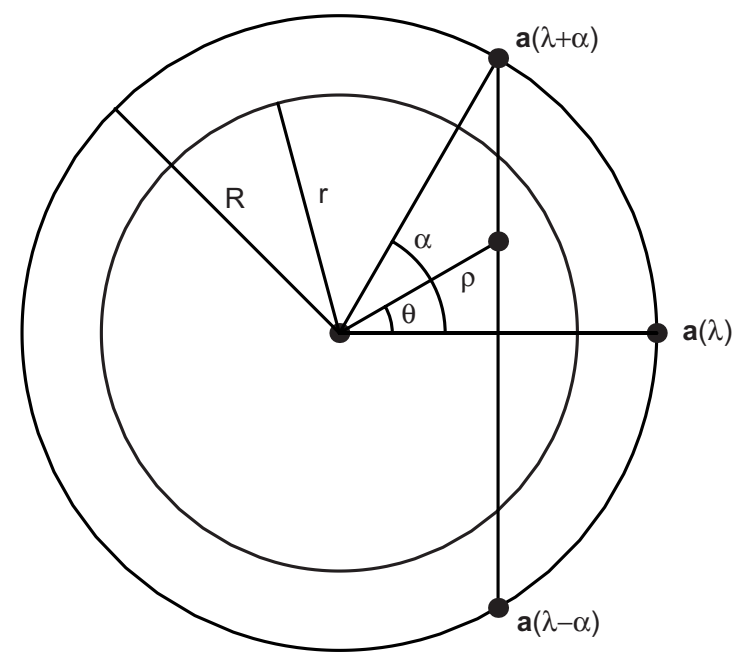

Figure 2. $C(\lambda)$ projected along the $z$ axis.

Definition 3. For $0 \leq \rho<1$, define $g_{\rho}:[-\pi, \pi] \rightarrow[-\pi, \pi]$ by

$$
g_{\rho}(\theta)=\theta-\frac{\rho \sin \theta \cos ^{-1}(\rho \cos \theta)}{\sqrt{1-\rho^{2} \cos ^{2} \theta}} .
$$

Since, $g_{\rho}$ is an odd function of $\theta$ and $g_{\rho}(0)=0$, it suffices to work with the interval $[0, \pi]$.

First, we show that $g_{\rho}$ is monotonically increasing.

Lemma 3. $g_{\rho}^{\prime}(\theta)>0$ on $[0, \pi]$.

Proof.

$$
g_{\rho}^{\prime}(\theta)=\frac{1-\rho^{2}}{\left(1-\rho^{2} \cos ^{2} \theta\right)^{3 / 2}}\left(\sqrt{1-\rho^{2} \cos ^{2} \theta}-\rho \cos \theta \cos ^{-1}(\rho \cos \theta)\right) .
$$

The leading fraction, $\left(1-\rho^{2}\right) /\left(1-\rho^{2} \cos ^{2} \theta\right)^{3 / 2}$, is positive. Substitute $z=\rho \cos \theta$ into the second term. Then $z \in[-\rho, \rho]$, and

$$
\sqrt{1-\rho^{2} \cos ^{2} \theta}-\rho \cos \theta \cos ^{-1}(\rho \cos \theta)=\sqrt{1-z^{2}}-z \cos ^{-1}(z) .
$$

Since

$$
\frac{d}{d z}\left(\sqrt{1-z^{2}}-z \cos ^{-1}(z)\right)=-\cos ^{-1} z,
$$

$\sqrt{1-z^{2}}-z \cos ^{-1}(z)$ is strictly decreasing on $[-1,1]$. Moreover,

$$
\left.\left(\sqrt{1-z^{2}}-z \cos ^{-1}(z)\right)\right|_{z=1}=0
$$

Hence, on $[-1,1)$, and in particular, on $[-\rho, \rho]$,

$$
\sqrt{1-z^{2}}-z \cos ^{-1}(z)>0 .
$$

Thus, the second term in (14) is also positive, and the lemma follows.

Lemma 4. $g_{\rho}^{-1}$ exists, and is continuous and differentiable on $[0, \pi]$. 
Proof. Since $\rho<1$, the denominator in the definition of $g_{\rho}$ does not vanish, so $g_{\rho} \in C^{\infty}([-\pi, \pi])$. For $\theta \in(0, \pi)$,

$$
\frac{\rho \sin \theta \cos ^{-1}(\rho \cos \theta)}{\sqrt{1-\rho^{2} \cos ^{2} \theta}}>0
$$

so $g_{\rho}(\theta)<\theta$. As $g_{\rho}$ is continuous and increasing, $g_{\rho}$ is injective. Since $g_{\rho}(0)=0$ and $g_{\rho}(\pi)=\pi$, the range is exactly $[0, \pi]$. Hence, $g_{\rho}^{-1}$ exists. As $g_{\rho}^{\prime} \neq 0$, the differentiability of $g_{\rho}^{-1}$ follows from the Inverse Function Theorem.

Remark 1. As $g_{\rho}$ is odd, Lemma 4 holds when the interval is extended to $[-\pi, \pi]$.

Lemma 5. Each point $\boldsymbol{x} \in S$ resides on exactly one chip.

Proof. Lemma 4 implies that (10) and (11) can be solved uniquely for $\theta \in(-\pi, \pi]$ and $\lambda \in \mathbb{R}$. Specifically, with $\beta \in(-\pi, \pi]$ given by

$$
\beta=\gamma-z / h \bmod 2 \pi,
$$

$\theta$, and $\lambda$ are recovered by

$$
\begin{aligned}
& \theta=g_{\rho}^{-1}(\beta), \\
& \lambda=\frac{z}{h}-\frac{\rho \sin \theta \cos ^{-1}(\rho \cos \theta)}{\sqrt{1-\rho^{2} \cos ^{2} \theta}} .
\end{aligned}
$$

Therefore, $\boldsymbol{x} \in C(\lambda)$.

As a consequence of Lemma 5 , the scan cylinder can be decomposed as a stack of chips. See Figure 3, As each chip is further decomposed into $\pi$-lines, a decomposition of the scan cylinder into a disjoint union of $\pi$-lines has been achieved. This is the substance of Theorem 1. Moreover, the geometric nature of this decomposition is now apparent. The above lemmas reduce the proof of Theorem 1 to only a few lines.

Proof of Theorem 1. Given $\boldsymbol{x} \in S$, determine $\theta$ and $\lambda$ as in (21) and (22) in the proof of Lemma 5. The corresponding $\alpha$ is found from the chip geometry:

$$
\alpha=\cos ^{-1}(\rho \cos \theta) .
$$

The unique $\pi$-line through $\boldsymbol{x}$ has endpoints $\boldsymbol{a}(\lambda-\alpha)$ and $\boldsymbol{a}(\lambda+\alpha)$.

\section{A FAST ALGORITHM FOR THE $\pi$-LINE ENDPOINTS}

In order to compute the $\pi$-line endpoints for a given $\boldsymbol{x} \in S$, it is necessary to implement (20)-(23). The difficulty is the computation of $g_{\rho}^{-1}$ in (21). In this section it is shown that Newton's method can be used for this computation and that there is at least a quadratic rate of convergence.

Theorem 6. Let $\beta \in[-\pi, \pi]$ be given. Then $g_{\rho}^{-1}(\beta)$ can be computed by Newton's method with quadratic convergence.

Proof. It suffices to prove the theorem for $\beta \in[0, \pi]$. Since $g_{\rho}^{-1}(0)=0$ and $g_{\rho}^{-1}(\pi)=$ $\pi$, we further restrict to $\beta \in(0, \pi)$.

Denote by $N g_{\rho}$ the Newton iteration

$$
N g_{\rho}(\theta)=\theta-\frac{g_{\rho}(\theta)-\beta}{g_{\rho}^{\prime}(\theta)}
$$




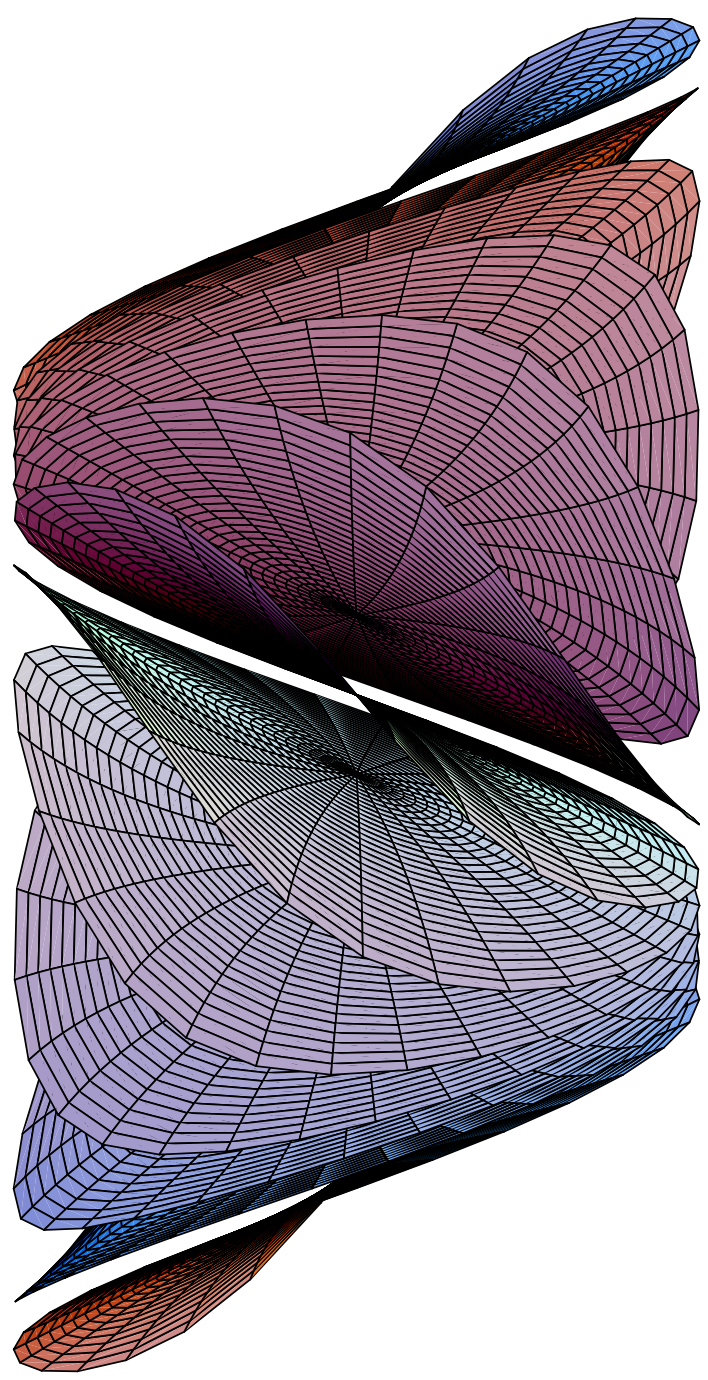

Figure 3. An illustration of the decomposition of the scan cylinder into a stack of chips. Several chips $C(\lambda)$ are shown, for $0 \leq \lambda \leq 2 \pi$. The pattern is periodic with period $2 \pi$, so the complete cylinder is obtained by vertical stacking of the translated copies of the region shown. As each chip is further decomposed into $\pi$-lines, the geometry of the decomposition of the cylinder into $\pi$-lines can be inferred from this figure. The figure was generated with parameters $R=1, r=3 / 4$, and $h=1 / 3$. 
$\theta_{0}=g_{\rho}^{-1}(\beta)$ if and only if $\theta_{0}$ is a fixed point for $N g_{\rho}$. Since $g_{\rho}$ is invertible, $N g_{\rho}$ has only one fixed point, and the theorem follows if it can be shown that $N g_{\rho}^{\prime}\left(\theta_{0}\right)=0$, and $N^{\prime} g_{\rho}(\theta) \gtrless 0$ for $\theta \lessgtr \theta_{0}[5]$.

A straightforward, but tedious, computation yields the derivative of the Newton iteration,

$$
N g_{\rho}^{\prime}(\theta)=\frac{N_{1} N_{2} N_{3}}{D_{1}}
$$

where

(26)

$$
\begin{aligned}
& N_{1}=\rho \sin \theta \\
& N_{2}=3 \rho \cos \theta \sqrt{1-\rho^{2} \cos ^{2} \theta}-\cos ^{-1}(\rho \cos \theta)\left(1+2 \rho^{2} \cos ^{2} \theta\right), \\
& N_{3}=(\beta-\theta) \sqrt{1-\rho^{2} \cos ^{2} \theta}+\rho \cos ^{-1}(\rho \cos \theta) \sin \theta, \\
& D_{1}=\left(-1+\rho^{2} \cos ^{2} \theta\right)\left(-1+\rho^{2} \cos ^{2} \theta+\rho \cos ^{-1}(\rho \cos \theta) \cos \theta \sqrt{1-\rho^{2} \cos ^{2} \theta}\right) .
\end{aligned}
$$

Substituting $z=\rho \cos \theta$ simplifies the expression for $N g_{\rho}^{\prime}$ to

$$
\begin{aligned}
& N_{1}=\sqrt{\rho^{2}-z^{2}} \\
& N_{2}=3 z \sqrt{1-z^{2}}-\left(1+2 z^{2}\right) \cos ^{-1} z, \\
& N_{3}=\sqrt{1-z^{2}}(\beta-\theta)+\sqrt{\rho^{2}-z^{2}} \cos ^{-1} z, \\
& D_{1}=\left(1-z^{2}\right)^{3 / 2}\left(\sqrt{1-z^{2}}-z \cos ^{-1} z\right) .
\end{aligned}
$$

As $z \in[-\rho, \rho], N_{1}>0$. The inequality (18) implies $D_{1}>0 . N_{2}<0$, because

$$
\frac{d N_{2}}{d z}=4\left(\sqrt{1-z^{2}}-z \cos ^{-1} z\right)>0
$$

and $N_{2}(1)=0$.

$$
\begin{aligned}
N_{3} & =\sqrt{1-z^{2}}\left(\beta-\theta+\frac{\sqrt{\rho^{2}-z^{2}} \cos ^{-1} z}{\sqrt{1-z^{2}}}\right), \\
& =\sqrt{1-z^{2}}\left(g_{\rho}\left(\theta_{0}\right)-g_{\rho}(\theta)\right) .
\end{aligned}
$$

As $g_{\rho}$ is monotonic increasing,

$$
N_{3} \gtreqless 0 \Longleftrightarrow \theta_{0} \gtreqless \theta,
$$

and therefore,

$$
N g_{\rho}^{\prime}(\theta) \gtreqless 0 \Longleftrightarrow \theta_{0} \lesseqgtr \theta .
$$

\section{ACKNOWLEDGEMENTS}

The author would like to thank David P. Rohler and K.L.A. Sastry for insightful discussions. 


\section{REFERENCES}

[1] P.E. Danielsson, P. Edholm, J. Eriksson, and M. Seger Magnusson, Toward exact reconstruction for helical cone-beam scanning of long objects. A new detector arrangement and a new completeness condition, Proc. 1997 Meeting on Fully 3D Image Reconstruction in Radiology and Nuclear Medicine, ed. D. W. Townsend and P. E. Kinahan, 1997, pp. 141-144.

[2] H. Turbell and P.E. Danielsson, An improved PI-method for reconstruction from helical conebeam projections, IEEE Nuclear Science Symposium, Conference Record, 2, 1999, pp. 865-868.

[3] M. Defrise, F. Noo, and H. Kudo, A solution to the long-object problem in helical cone-beam tomography, Phys. Med. Biol. 45, 2000, pp. 623-643.

[4] A. Katsevich, Improved Exact FBP Algorithm for Spiral CT, Advances in Applied Mathematics, 32, 2004, pp. 681-697. MR.2053840 (2005b:44002)

[5] J. Stoer and R. Bulirsch, Introduction to Numerical Analysis, Springer-Verlag, New York, 1980. MR0557543(83d:65002)

Department of Mathematics, Case Western Reserve University, Cleveland, Ohio 44106

E-mail address: shi@cwru.edu 